\title{
Transient Hypoxia Alters Striatal Catecholamine Metabolism in Immature Brain: An In Vivo Microdialysis Study
}

\author{
${ }^{*}+$ Kevin Gordon, ${ }^{*}+$ Daniel Statman, ${ }^{*}+$ Michael V. Johnston, + Terry E. Robinson, \\ $\ddagger$ Jill B. Becker, and $*+$ Faye S. Silverstein
}

Departments of *Pediatrics, $\uparrow$ Neurology, and $\ddagger$ Psychology, University of Michigan, Ann Arbor, Michigan, U.S.A.

\begin{abstract}
Microdialysis probes were inserted bilaterally into the striatum of 7-day-old rat pups $(n=30)$ to examine extracellular fluid levels of dopamine, its metabolites 3,4-dihydroxyphenylacetic acid (DOPAC) and homovanillic acid (HVA), and the serotonin metabolite 5-hydroxyindoleacetic acid (5-HIAA). The dialysis samples were assayed by HPLC with electrochemical detection. Baseline levels, measured after a 2-h stabilization period, were as follows: dopamine, not detected; DOPAC, $617 \pm 33 \mathrm{fmol} / \mathrm{min}$; HVA, $974 \pm 42 \mathrm{fmol} /$ $\mathrm{min}$; and 5-HIAA, $276 \pm 15 \mathrm{fmol} / \mathrm{min}$. After a $40-\mathrm{min}$ baseline sampling period, 12 animals were exposed to $8 \%$ oxygen for $120 \mathrm{~min}$. Hypoxia produced marked reductions in the striatal extracellular fluid levels of both dopamine metabolites ( $p<0.001$ by analysis of variance) and a more gradual and less prominent reduction in 5-HIAA levels $(p<0.02$ by analysis of variance), compared with controls $(n=12)$ sampled
\end{abstract}

in room air. In the first hour after hypoxia, DOPAC and HVA levels rose quickly, whereas 5-HIAA levels remained suppressed. The magnitude of depolarization-evoked release of dopamine (elicited by infusion of potassium or veratrine through the microdialysis probes for $20 \mathrm{~min}$ ) was evaluated in control and hypoxic animals. Depolarization-evoked dopamine efflux was considerably higher in hypoxic pups than in controls: hypoxic $(n=7), 257 \pm 32 \mathrm{fmol} / \mathrm{min}$; control ( $\mathrm{n}$ $=12), 75 \pm 14 \mathrm{fmol} / \mathrm{min}(p<0.001$ by analysis of variance $)$. These data demonstrate that a brief exposure to moderate hypoxia markedly disrupts striatal catecholamine metabolism in the immature rodent brain. Key Words: Dopamine-Microdialysis-Striatum-Immature brain-Hypoxia. Gordon K. et al. Transient hypoxia alters striatal catecholamine metabolism in immature brain: An in vivo microdialysis study. J. Neurochem. 54, 605-611 (1990).
In the perinatal period, infants with various clinical disorders are commonly exposed to brief, as well as more prolonged, periods of hypoxia. The immature brain is considered to be relatively resistant to neuropathologic damage from moderate hypoxia, and it is uncertain if moderate hypoxia, e.g., $\mathrm{P}_{\mathrm{A}} \mathrm{O}_{2}$ of 25-50 torr, alone can produce either acute behavioral changes or long-term adverse neurodevelopmental sequelae. Nevertheless, exposure to moderate hypoxia during early postnatal life, which does not cause neuronal damage, may disrupt functional activity at selected synapses in experimental animals (Hedner and Lundborg, 1979, 1980). Furthermore, there is evidence that exposure to hypoxia at critical stages in development may cause permanent changes in synaptic function (Ihle et al., 1985; Lun et al., 1986).
Studies in mature animals indicate that dopaminergic neurons are susceptible to functional disruption by moderate hypoxia (Davis and Carlsson, 1973; Miwa et al., 1986). Less is known about the effects of hypoxia on developing dopaminergic neurons, but the 7-dayold rat has a potentially functional dopaminergic innervation in the striatum. In addition, we reported previously that hypoxia transiently suppressed both tissue concentrations of the dopamine metabolite homovanillic acid (HVA) and dopamine uptake into striatal tissue prepared from immature rodent brain (Silverstein and Johnston, 1984; Silverstein et al., 1986).

In this study, we used in vivo microdialysis to examine the effects of hypoxia on dopamine metabolism more directly, by measuring striatal extracellular fluid
Received March 1, 1989; revised manuscript received July 6, 1989; accepted July 7, 1989.

Address correspondence and reprint requests to Dr. F. S. Silverstein at Neuroscience Lab Building, 1103 East Huron, Ann Arbor, MI 48104-1687, U.S.A.

The present address of Dr. K. Gordon is Dalhousie University, Halifax, Nova Scotia, Canada.
The present address of Dr. M. V. Johnston is Kennedy Institute, Johns Hopkins University, Baltimore, MD 21218, U.S.A.

Abbreviations used: DOPAC, 3,4-dihydroxyphenylacetic acid; ECF, extracellular fluid; 5-HIAA, 5-hydroxyindoleacetic acid; HPLC-EC, HPLC with electrochemical detection; HVA, homovanillic acid; $\mathrm{MAO}$, monoamine oxidase. 
(ECF) concentrations of dopamine and its metabolites as well as of the serotonin metabolite 5-hydroxyindoleacetic acid (5-HIAA) before, during, and after exposure to $8 \%$ oxygen. In addition, we evaluated changes in depolarization-induced dopamine release and striatal tissue concentrations of dopamine after hypoxic exposure.

We found that hypoxic exposure resulted in marked suppression of striatal ECF levels of the dopamine metabolites 3,4-dihydroxyphenylacetic acid (DOPAC) and HVA and a more gradual and less prominent reduction in levels of the serotonin metabolite 5-HIAA. After the end of hypoxic exposure, DOPAC and HVA levels rose quickly, whereas 5-HIAA levels remained suppressed. In addition, the magnitude of depolarization-evoked dopamine release (elicited by infusion of potassium or veratrine) was considerably higher in hypoxic pups than in controls, and there was a corresponding elevation in striatal dopamine concentrations measured in postmortem tissue.

\section{MATERIALS AND METHODS}

\section{Experimental design}

Microdialysis probes were implanted bilaterally into the striatum of 7-day-old male Sprague-Dawley rat pups (n $=54$ ). Subsequent histological examination (see below) verified accurate, symmetrical probe placement in 30 pups $(57 \%)$, and only data from these pups are presented. The protocol included (a) probe insertion and stabilization (210 $\mathrm{min}$ ); (b) microdialysis sampling over 20 -min intervals during the three sequential periods of baseline (40 $\mathrm{min})$, experimental (160 $\mathrm{min}$ ), and recovery (60 $\mathrm{min}$ ); and (c) assay of depolarizationevoked dopamine release by infusion of depolarizing agents $\left(100 \mathrm{mM} \mathrm{K} \mathrm{K}^{+}\right.$or $100 \mu \mathrm{g} / \mathrm{ml}$ of veratrine) over a $20-\mathrm{min}$ period at the end of the baseline and experimental periods. From animals that received infusions of depolarizing drugs, no subsequent samples were obtained. Results from two additional pups were included in a single component of this study, analysis of depolarization-evoked dopamine release after hypoxic exposure.

During the experimental period, animals were exposed to $8 \%$ oxygen $(n=12)$ or room air $(n=12)$. Previously, we found that in awake 7-day-old rat pups, exposure to $8 \%$ oxygen results in an abrupt and sustained decline in arterial oxygen content $\left(\mathrm{P}_{\mathrm{A}} \mathrm{O}_{2}, 30\right.$ torr $)$ and concurrently elicits hyperventilation $\left(\mathrm{P}_{\mathrm{A}} \mathrm{CO}_{2}, 25\right.$ torr) (Silverstein and Johnston, 1984). This degree of hypoxia does not result in global energy failure in brain (Welsh et al., 1982) but is of sufficient consequence to suppress striatal high-affinity dopamine uptake (Silverstein et al., 1986). To evaluate depolarization-evoked ECF dopamine efflux, control pups received either $100 \mathrm{mM}$ $\mathrm{K}^{+}(\mathrm{n}=3)$ or $100 \mu \mathrm{g} / \mathrm{ml}$ of veratrine $(\mathrm{n}=3)$ after the baseline period, and another sample was obtained. At the end of the experimental period, hypoxic pups $(\mathbf{n}=7)$ were perfused with either $100 \mathrm{mM} \mathrm{K} \mathrm{K}^{+}(\mathrm{n}=2)$ or $100 \mu \mathrm{g} / \mathrm{ml}$ of veratrine ( $=5$ ) for $20 \mathrm{~min}$, and a final sample was obtained. The control animals received infusions of $\mathrm{K}^{+}(\mathrm{n}=3)$ or veratrine $(\mathrm{n}$ $=3$ ) during this same interval. Of note was that $100 \mathrm{~m} M \mathrm{~K}^{+}$ was infused to ensure that the $\mathrm{K}^{+}$concentrations achieved in brain were adequate to induce depolarization; $\mathrm{K}^{+}$concentrations in brain were probably considerably lower because

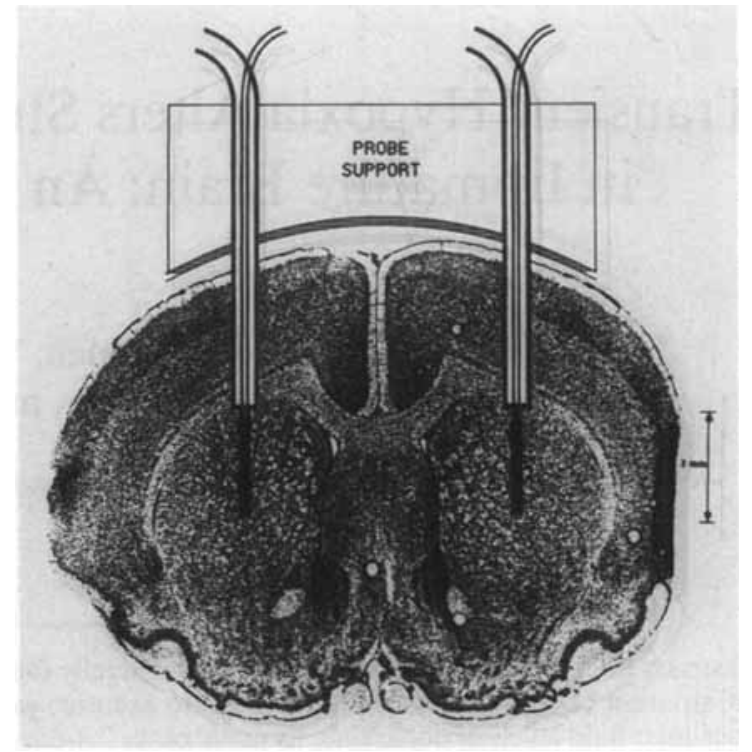

FIG. 1. Illustration of the sites of bilateral probe placement. The sites of probe insertion are superimposed on a coronal brain section from a 7 -day-old rat, at the level of striatum.

of the rate of diffusion through the probe membrane as well as dilution by the ECF.

\section{Microdialysis probe design}

The microdialysis probe design was a modification of that described previously by Robinson and Whishaw (1988). The probes had a concentric design with a 2-mm-long dialyzing membrane (outside diameter, $250 \mu \mathrm{m}$; molecular-weight cutoff, 6,000; Spectra/Por; Spectrum Medical). Microdialysis probe efficiency was calculated for each of the compounds detected in vitro at $37^{\circ} \mathrm{C}$ using the average of two samples (Ungerstedt, 1984). The relative recovery values obtained (mean \pm SEM, $\mathbf{n}=60$ ) were as follows: dopamine, 21.6 $\pm 0.5 \%$; DOPAC, $21.3 \pm 0.4 \%$; HVA, $20 \pm 0.4 \%$; and $5-$ HIAA, $21.8 \pm 0.5 \%$.

Two microdialysis probes were placed in a rigid plastic mounting system that could be readily affixed to the skull surface (Fig. 1). The mounting system was constructed from a plastic slide holder with two parallel channels $(2 \mathrm{~mm}$ wide, $3 \mathrm{~mm}$ deep, and $5 \mathrm{~mm}$ apart). The mounting surface was carved concave to conform to the shape of the skull and was then cut in half. The microdialysis probes were glued (Devcon 5 Minute Epoxy) to the upper section.

\section{Microdialysis probe insertion}

Rat pups were anesthetized with ether. An oval scalp flap $16 \mathrm{~mm}$ (anteroposterior) by $9 \mathrm{~mm}$ (lateral), centered on bregma, was excised. Pups were then left under a heating lamp and a stream of warm air for $90 \mathrm{~min}$ to dry the skull surface, thus ensuring adhesion of the microdialysis probe mount to the skull. The pups were then reanesthetized with ether and placed in a stereotaxic frame. The concave section of the probe mount was aligned at bregma and attached to the skull with cyanoacrylate glue (Superglue). Bilateral cranial flaps $(2 \times 2 \mathrm{~mm})$ were removed at the base of the channels of the lower probe mount. The upper section of the probe mount holding the two parallel microdialysis probes was then lowered with stereotactic guidance, allowing simultaneous 
implantation of the microdialysis probes into striatum when the two sections of the probe mount met. The two sections of the probe mount were reattached with cyanoacrylate glue (Superglue). The exposed skull surface and the edges of the lower probe mount were coated with a thin layer of epoxy (Devcon 5 Minute). The final position of the microdialysis membrane was $1 \mathrm{~mm}$ posterior and $2.5 \mathrm{~mm}$ lateral to bregma and 3-5 $\mathrm{mm}$ from the skull surface (Fig. 1). The implantation of the probes was completed within 5 min. Pups were then placed in a plastic chamber heated in a $34^{\circ} \mathrm{C}$ water bath.

At the end of each experiment, pups were killed by decapitation, and brains were quickly removed and placed in formalin. Microdialysis probe placement was subsequently verified by examination of horizontal brain sections. Blood glucose levels, measured by Dextrostix on blood obtained from the carotid arteries, were normal in all pups.

\section{Sample collection}

Microdialysis probes were perfused with filtered modified Ringer's solution ( $147.2 \mathrm{mmol}$ of $\mathrm{Na}^{+}, 4.0 \mathrm{mmol}$ of $\mathrm{K}^{+}, 3.4$ mmol of $\mathrm{Ca}^{2+}$, and $154.6 \mathrm{mmol}$ of $\mathrm{Cl}^{-}, \mathrm{pH} 6.0$ ) at $1.5 \mu \mathrm{l} /$ min using a microinfusion pump (CMA 100; BAS).

No samples were collected for the first 120 min after implantation. Samples were then collected on ice over successive 20-min intervals. The first two samples (baseline period; 40 min) were used to estimate baseline ECF neurotransmitter and metabolite levels. During the next seven samples (experimental period; $140 \mathrm{~min}$ ), animals were exposed to control or hypoxic conditions. The three final samples were obtained in room air (recovery period; $60 \mathrm{~min}$ ).

In animals exposed to hypoxic conditions, the atmospheric oxygen concentration in the chamber was measured at regular intervals with a Beckman oxygen analyzer, to maintain the oxygen concentration at $8 \%$. As well, in selected animals, surface temperature was monitored throughout the experiment with an electronic thermometer; surface temperature from the time of probe insertion remained stable $\left(34^{\circ} \mathrm{C}\right)$ for the duration of hypoxic exposurc.

Stimulated dopamine release was measured in control animals $(\mathrm{n}=6)$ during the first $20 \mathrm{~min}$ following the baseline period. Stimulated dopamine release was also measured in both control $(n=6)$ and hypoxic $(n=7)$ rats during the 20 min interval following the experimental period. During stimulated release periods, the probes were perfused with $100 \mu \mathrm{g} /$ $\mathrm{ml}$ of veratrine (Sigma) dissolved in the modified Ringer's solution or that containing $100 \mathrm{~m} M \mathrm{~K}^{+}\left(51.2 \mathrm{mM} \mathrm{Na}{ }^{+}, 100.0\right.$ $\mathrm{m} M \mathrm{~K}^{+}, 3.4 \mathrm{~m} M \mathrm{Ca}^{2+}$, and $154.6 \mathrm{mMCl}^{-}, \mathrm{pH} \mathrm{6.0)}$.

\section{Histology}

Brains were fixed in formalin, and 12-16 horizontal 50$\mu \mathrm{m}$-thick sections from each brain were stained with cresyl violet. The probe tips produced a readily identifiable anatomic defect. Sections were independently scored by two observers for symmetry and accuracy of placement; only data from rat pups where there was agreement between the observers were analyzed. The most commonly observed exclusion criteria were ventricular dilatation, inability to identify the placement sites, and placement into globus pallidus.

\section{Neurotransmitter and metabolite levels in striatal tissue}

The effect of hypoxic exposure on the postmortem striatal tissue content of dopamine, HVA, DOPAC, and 5-HIAA was also measured. Seven-day-old male Sprague-Dawley rat pups were placed in plastic chambers within a $34^{\circ} \mathrm{C}$ water bath and exposed to hypoxic ( $8 \%$ oxygen) or control conditions ( $n=6 /$ group) for $150 \mathrm{~min}$. Pups were killed by decapitation, and the striata were dissected out on ice, frozen, and stored at $-70^{\circ} \mathrm{C}$.

\section{HPLC with electrochemical detection}

Dialysate samples were analyzed within $20 \mathrm{~min}$ after collection by HPLC with electrochemical detection (HPLC-EC) Twenty-microliter microdialysis samples were injected directly onto a $\mathrm{C}-18$ reverse-phase Ultrasphere column $(15 \mathrm{~cm}$ $\times 4.6 \mathrm{~mm}$ inner diameter; $5 \mu \mathrm{m}$ ODS; Beckman) using a WISP $710 \mathrm{~B}$ autoinjector. The mobile phase contained 0.1 $M$ sodium citrate, $0.1 M$ sodium phosphate monobasic, 0.1 $\mathrm{m} M$ EDTA, and $22.5 \%$ methanol, adjusted to $\mathrm{pH} 3.5$. Sodium octyl sulfate was titrated to adjust the retention time of dopamine, with a final concentration of $\sim 2.15 \mathrm{~m} M$. Detection was by a glassy carbon electrode connected to an electrochemical detector (LC4A Amperometric Detector; Bioanalytical Systems), with a sensitivity of $1-2 \mathrm{nA}$ and an electrode potential of $0.76 \mathrm{~V}$. Detector output was recorded on a chart recorder, peak heights were measured, and absolute amounts were calculated by comparison with peak heights of standards included in each assay. The detection thresholds of the assay (defined as peak heights greater than twice baseline fluctuations) were as follows: dopamine, $36 \mathrm{fmol}$; DOPAC, $32 \mathrm{fmol}$; HVA, 81 fmol; and 5-HIAA, 24 fmol. Serotonin was not quantified in the dialysis samples.

In preliminary experiments, we examined the stability of dopamine and the metabolite standards in the Ringer's solution used for dialysis. We found that freezing and subsequent thawing of samples reduced the reliability of dopamine content measurements. In fresh samples, placed on ice, there was no deterioration in peak heights in aliquots sampled over several hours. To optimize the assay, all dialysis samples were assayed within 20 min after collection.

To measure striatal tissue levels of dopamine, DOPAC, HVA, serotonin, and 5-HIAA, frozen striatal tissue was homogenized in $400 \mu \mathrm{l}$ of perchloric acid and briefly centrifuged, and extracts were analyzed by the same HPLC-EC assay (modified after Zaczek and Coyle, 1982).

\section{Data analysis}

Dialysate values (mean \pm SEM), corrected for probe recovery, are expressed as femtomoles per minute. Tissue concentrations are expressed as picomoles per microgram of wet tissue weight. Descriptive statistics, analyses of variance, and $t$ tests were performed using a microcomputer-based statistical package (Systat, Evanston, IL, U.S.A.).

\section{RESULTS}

Microdialysis probes were implanted bilaterally because of potential striatal dopaminergic asymmetries (Fig. 1). Histological examination confirmed accurate, symmetrical microdialysis probe placement in the striatum in $57 \%$ of the animals. Only data from these pups were included for analysis. Preliminary analysis of basal valucs of metabolites did not demonstrate any interhemispheric asymmetries. Therefore, all data presented represent averages of the left and right sides.

DOPAC, HVA, and 5-HIAA were detected in all samples. Preliminary experiments demonstrated that the ECF concentrations of DOPAC, HVA, and 5HIAA were depressed during the first $20 \mathrm{~min}$ after in- 


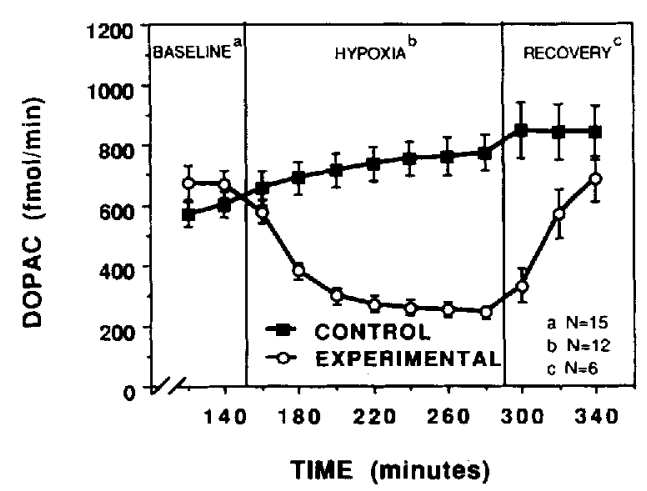

FIG. 2. Changes in striatal ECF concentrations of DOPAC during hypoxic exposure in 7-day-old rats. Microdialysis sampling was initiated $2 \mathbf{h}$ after probe insertion (see Materials and Methods), and samples were collected over 20-min intervals. The protocol included a baseline period (40 min), a 140-min period during which animals were exposed to $8 \%$ oxygen (experimental) or room air (controls), and a 60-min recovery period in room air. Data, corrected for probe recovery, are mean \pm SEM (bars) values. $p<0.001$ by repeatedmeasures analysis of variance.

sertion of the microdialysis probes (DOPAC, 166.6 $\pm 31.2 \mathrm{fmol} / \mathrm{min} ; \mathrm{HVA}, 338.3 \pm 59.7 \mathrm{fmol} / \mathrm{min}$; and 5-HIAA, $142.9 \pm 25.4 \mathrm{fmol} / \mathrm{min} ; \mathrm{n}=6$ ). Metabolite levels progressively increased over the next $100 \mathrm{~min}$ and stabilized, i.e., the change between sequential samples was $<5 \%$, at $120-160$ min after probe insertion. Based on these preliminary observations, baseline sample collection was initiated after a 120 -min stabilization period.

Effects of hypoxia on ECF metabolite concentrations

During the baseline period, ECF metabolite concentrations did not significantly differ between the control and experimental rat pups. Baseline values (averaged from control and experimental animals; $n=30$ ) were as follows: DOPAC, $617.2 \pm 33.1 \mathrm{fmol} / \mathrm{min}$; HVA, $974.0 \pm 41.5 \mathrm{fmol} / \mathrm{min}$; and 5-HIAA, $276.0 \pm 14.6$ $\mathrm{fmol} / \mathrm{min}$. Over the experimental and recovery periods

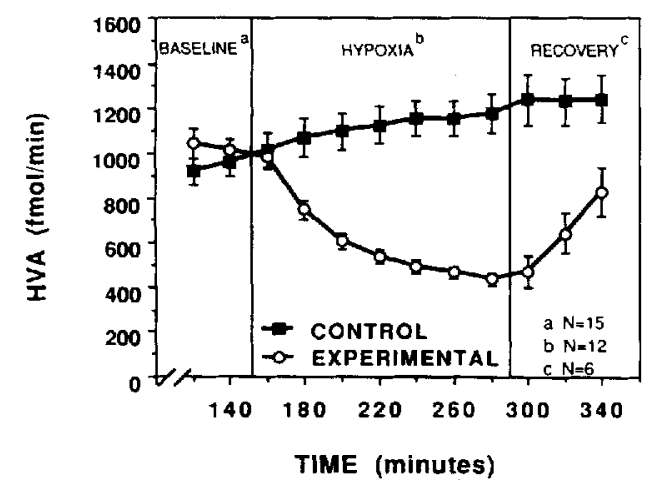

FIG. 3. Changes in striatal ECF concentrations of HVA during hypoxic exposure in 7-day-old rats. See the legend of Fig. 2 for a description of the experimental protocol. Data, corrected for probe recovery, are mean \pm SEM (bars) values. $p<0.001$ by repeatedmeasures analysis of variance.

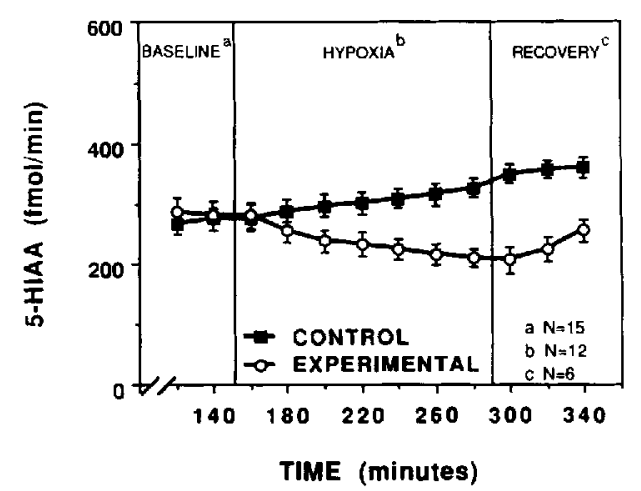

FIG. 4. Changes in striatal ECF concentrations of 5-HIAA during hypoxic exposure in 7-day-old rats. See the legend of Fig. 2 for a description of the experimental protocol. Data, corrected for probe recovery, are expressed as mean \pm SEM (bars) values. $p=0.012$ by repeated-measures analysis of variance.

(200 min), metabolite concentrations continued to increase in control animals to $130 \pm 8 \%$ for DOPAC (Fig. 2), $127 \pm 8 \%$ for HVA (Fig. 3), and $131 \pm 13 \%$ for 5-HIAA (Fig. 4) of baseline values $(n=6)$.

In contrast, hypoxia produced a marked decrease in the ECF concentrations of DOPAC, HVA, and 5HIAA (Fig. 2-4) when compared with controls (DOPAC, $p<0.001$; HVA, $p<0.001$; and 5-HIAA, $p$ $=0.019$; by repeated-measures analysis of variance). The DOPAC content declined rapidly to $48.0 \pm 4.4 \%$ of basal values within $60 \mathrm{~min}$ of hypoxia (Fig. 2) and had leveled off at $39.5 \pm 3.6 \%$ of baseline values by the end of the period of hypoxic exposure $(140 \mathrm{~min})$. The temporal pattern and magnitude of depression of HVA levels during hypoxia were similar to those of DOPAC levels; HVA concentrations fell to $44.5 \pm 3.1 \%$ of baseline values by the end of hypoxic exposure (Fig. 3). In contrast, Fig. 4 shows that ECF concentrations of 5HIAA declined more slowly and to a lesser degree than did the levels of the dopamine metabolites during the period of hypoxia (to $75.5 \pm 5.6 \%$ of baseline).

During the 60 -min recovery period in room air, concentrations of both DOPAC and HVA returned rapidly toward control values (DOPAC, $p<0.001$; HVA, $p<0.001$; by Student's $t$ test comparing values at the end of hypoxia with those at the end of the recovery period). By the last sample interval, control and hypoxic pups did not differ significantly in their levels of DOPAC, but in posthypoxic pups the HVA content remained lower than in controls $(p=0.02$ by Student's $t$ test). In contrast, 5-HIAA concentrations in previously hypoxic rat pups remained relatively depressed during the recovery period.

\section{Effects of hypoxia on ECF dopamine}

In the adult rat striatum, reported baseline striatal ECF dopamine values range from $\sim 60$ to $110 \mathrm{fmol} /$ min (Zetterstrom et al., 1983; Westerink et al., 1984; Sharp et al., 1986; Robinson and Whishaw, 1988). The calculated threshold for detection of ECF dopamine 


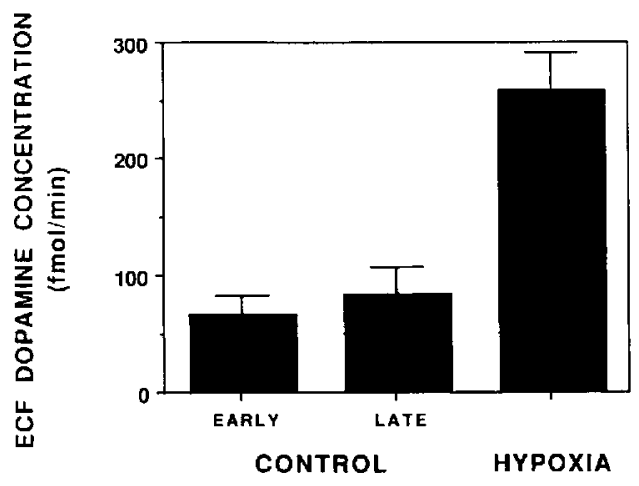

FIG. 5. Comparison of the magnitude of depolarization-evoked dopamine release in control and hypoxic pups. Infusion of the depolarizing agents - potassium $(100 \mathrm{mM})$ or veratrine $(100 \mu \mathrm{g} / \mathrm{ml})$ was carried out at $160-180$ min (EARLY; $n=6$ ) or at $300-320$ $\min ($ LATE; $n=6$ ) after probe insertion in controls and after the conclusion of $140 \mathrm{~min}$ of exposure to $8 \%$ oxygen (300-320 min after probe insertion; $n=7$ ) in the experimental group. $p=0.001$ by analysis of variance.

with our assay methods was $8 \mathrm{fmol} / \mathrm{min}$ (based on a detection threshold of $36 \mathrm{fmol} / 20-\mu \mathrm{l}$ sample and a recovery efficiency of $21.6 \%$ ). In preliminary experiments using 7-day-old pups, dopamine was not detected during the 120 min following probe insertion $(n=6)$. In the hypoxia group, dopamine was detected during the basal period in one animal $(9.4 \mathrm{fmol} / \mathrm{min})$. Dopamine was not detected in any of the 12 control rat pups during the experimental period (160-300 min). In contrast, dopamine was detected, in at least one sample, in six of 12 rat pups during hypoxic exposure. Thus, during the experimental period dopamine was detected in significantly more animals exposed to hypoxic conditions than in control animals: none of 12 versus six of 12 , respectively ( $p=0.014$ by Fisher's exact test).

\section{Stimulated dopamine release}

Potassium and veratrine consistently stimulated dopamine accumulation in microdialysis samples (Fig. 5). There were no differences between responses elicited from left and right sides or between the responses to these doses of potassium and veratrine. Therefore, values from left and right striata were averaged, and values from potassium- and veratrine-stimulated release were pooled.

In controls, the timing of the potassium or veratrine infusion [160-180 $\min (\mathrm{n}=6)$ vs. $300-320 \mathrm{~min}(\mathrm{n}$ $=6$ ) following implantation] did not alter the magnitude of dopamine release. The averaged value for depolarization-evoked ECF dopamine efflux in all controls was $74.7 \pm 13.9 \mathrm{fmol} / \mathrm{min}(\mathrm{n}=12)$. However, Fig. 5 shows that potassium or veratrine infusion after $140 \mathrm{~min}$ of $8 \%$ oxygen exposure $(n=7)$ increased ECF dopamine concentrations to $344 \%$ of the control levels $(257.1 \pm 32.1 \mathrm{fmol} / \mathrm{min} ; p<0.001$ by analysis of variance). No significant differences in metabolite concentrations were observed between control and hypoxic animals during this period.

\section{Effects of hypoxia on tissue concentration of dopamine}

In striatal tissue, there were corresponding increases in dopamine content after hypoxia (Table 1). In pups killed immediately after 150 min of hypoxia, striatal dopamine concentrations were $14.70 \pm 0.62 \mathrm{pmol} / \mathrm{mg}$ of tissue, compared with $11.36 \pm 0.94 \mathrm{pmol} / \mathrm{mg}$ of tissue in controls ( $p=0.014$ by Student's $t$ test). The postmortem tissue concentrations of serotonin, its metabolite 5-HIAA, and the dopamine metabolites DOPAC and HVA were not altered by hypoxia (Table 1).

\section{DISCUSSION}

These data demonstrate that in perinatal rats exposure to moderate hypoxia produced large alterations in striatal catecholamine metabolism. The ECF concentrations of dopamine and serotonin metabolites were suppressed acutely, whereas dopamine itself was more frequently detected in the ECF. After $2 \mathrm{~h}$ of hypoxia, both depolarization-evoked dopamine release and tissue dopamine content rose. Comparison of the magnitudes and temporal patterns for depression and subsequent recovery of the ECF concentrations of the dopamine and serotonin metabolites indicates differential effects of hypoxia on these two neuronal populations.

During exposure to hypoxia, concentrations of HVA, DOPAC, and 5-HIAA declined. Reduced striatal ECF concentrations of neurotransmitter metabolites could reflect changes in the production or removal of the metabolites or of the parent neurotransmitters (decreased synthesis or release, increased uptake or removal, or both).

The activity of the degradative enzymes monoamine oxidase (MAO) and catechol- $O$-methyl transferase may be limited in vivo at the level of hypoxia that was examined, and decreased degradation rates could, in part, account for the observed reductions in HVA and DOPAC levels. Serotonin is also metabolized by MAO to 5-HIAA; 5-HIAA did not show a depression in ECF concentrations equivalent to that of DOPAC or HVA

TABLE 1. Effects of hypoxia on striatal concentrations of dopamine, serotonin, and their metabolites

\begin{tabular}{lcc}
\hline & \multicolumn{2}{c}{ Concentration $(\mathrm{pmol} / \mathrm{mg}$ of tissue) } \\
\cline { 2 - 3 } Compound & Control & Hypoxia \\
\hline Dopamine & $11.36 \pm 0.94$ & $14.70 \pm 0.62^{a}$ \\
DOPAC & $2.26 \pm 0.41$ & $1.97 \pm 0.31$ \\
HVA & $1.89 \pm 0.22$ & $1.87 \pm 0.48$ \\
Serotonin & $0.94 \pm 0.04$ & $0.97 \pm 0.07$ \\
5-HIAA & $0.80 \pm 0.08$ & $0.66 \pm 0.08$ \\
\hline
\end{tabular}

Seven-day-old male rat pups were exposed to $8 \%$ oxygen $(n=6)$ or room air $(n=6)$ for 150 min. Animals were then killed by decapitation, and striata were quickly removed. Neurotransmitter levels were assayed by HPLC-EC in perchloric acid extracts.

${ }^{a}$ In animals exposed to hypoxia, tissue concentrations of dopamine were higher than in controls $(p=0.014$ by $t$ test). 
during hypoxia, but this could conceivably reflect more efficient reuptake of serotonin than dopamine and the availability of a higher concentration of substrate for MAO. In contrast, more efficient removal of metabolites from the ECF is unlikely to account for the pattern of reduction in metabolite concentrations observed, because the three metabolites are carried out of the ECF by a common transporter. Similarly, changes in cerebral blood flow are unlikely to account for the decreased metabolite levels. Results of two studies indicate that exposure to $8 \%$ oxygen does not augment cerebral blood flow in 7-day-old rats. Quantitation of regional cerebral perfusion by measurement of accumulation of tracer in cortex and striatum after intracardiac injection of $\left[{ }^{14} \mathrm{C}\right]$ iodoantipyrine or $\left[{ }^{3} \mathrm{H}\right]$ flunitrazepam demonstrated no increase in tracer accumulation during hypoxia (Silverstein et al., 1984). Similarly, measurement of regional cerebral blood flow in 7-day-old rats after unilateral carotid ligation and exposure to $8 \%$ oxygen revealed no change in flow to the contralateral hemisphere during hypoxia (Vannucci et al., 1988). Furthermore, any increased washout of metabolites as a result of increased blood flow would be expected to affect dopamine and serotonin metabolites to the same extent.

The depolarization data provide information about the effects of hypoxia on dopamine itself. The increase in depolarization-evoked release could result from the intracellular translocation of dopamine to a releasable pool. However, the elevations in tissue dopamine levels indicate that total intracellular dopamine content is increased. These changes could reflect decreased release, decreased intracellular degradation, increased synthesis, or increased reuptake of dopamine during hypoxia. Available data suggest that decreased dopamine release, together perhaps with decreased intracellular degradation, most likely accounts for our observations.

Increased reuptake of released dopamine could also result in intraneuronal dopamine accumulation. However, assays of $\left[{ }^{3} \mathrm{H}\right]$ dopamine uptake in synaptosomal preparations from the striatum of 7-day-old rats exposed to $8 \%$ oxygen for $2 \mathrm{~h}$ demonstrate suppression of uptake (Silverstein et al., 1986). Thus, reduced reuptake activity may well account for the increased detection frequency of dopamine in ECF during hypoxia that we observed.

Several issues must be considered in the overall interpretation of these results in immature rodent brain. Benveniste et al. (1987) reported that probe insertion acutely disrupts hippocampal glucose metabolism and blood flow in mature animals (most pronounced in the first $2 \mathrm{~h}$ ) and recommended a prolonged stabilization period before initiation of dialysis sampling. A major limitation of microdialysis in immature rodents is their dependence on the dam for nourishment. Because pups cannot be returned to the dam after probe insertion, the inclusion of a more prolonged stabilization period is not feasible. In the rat pups, the baseline was rela- tively stable when sampling was initiated $2 \mathrm{~h}$ after probe insertion. During the course of the experiments, in controls, the concentrations of neurotransmitter metabolites continued to increase slowly; the factors contributing to this gradual rise are uncertain. Differences in the magnitudes and temporal patterns of metabolite suppression and the subsequent recovery between dopamine metabolites and 5-HIAA were apparent. This specificity suggests that it is unlikely that the results reflect systemic derangements, e.g., temperature instability or dehydration. However, recent studies indicate that small reductions in brain temperature can significantly alter neurotransmitter release (Globus et al., 1989). Because intracranial temperature was not measured directly in the rat pups during hypoxic exposure, hypothermia cannot be excluded as a factor contributing to the suppression of metabolite levels.

There is considerable controversy about the accuracy with which ECF metabolite levels reflect the rate of dopamine release. Although DOPAC and HVA may be useful indices of central dopamine release (Roth et al., 1976), inconsistencies in the relationship between levels of dopamine and its metabolites in dialysate samples have been reported (Sharp et al., 1986). These issues have not been examined directly in the immature brain. Furthermore, there is no information about changes in the relative roles of reuptake and enzymatic degradation of released dopamine during development.

In the assays of striatal tissue, no reductions in dopamine or serotonin metabolite concentrations were observed after hypoxia. Previously, we had reported that HVA and 5-HIAA levels declined in striatal tissue during hypoxia (Silverstein and Johnston, 1984); the differences between the two sets of results could conceivably reflect the somewhat longer period of hypoxia used and the restriction to male animals in these experiments. Although tissue neurotransmitter levels represent the combined extra- and intraneuronal concentrations, the observed discrepancy between dialysate and tissue levels of metabolites suggests that tissue levels reflect intraneuronal concentrations to a greater extent and that these are differences in the susceptibility to suppression by hypoxia of intra- and extraneuronal degradative enzymes. Similarly, the differences in relative ratios of HVA and DOPAC (HVA:DOPAC ratio, 1.58 in ECF and 0.84 in tissue) may reflect differences between intra- and extraneuronal metabolism. There is little information about developmental changes in dopamine catabolism. However, our observations that ECF levels of HVA are $~ 50 \%$ higher than DOPAC levels in the 7-day-old rat, whereas in adult animals the ratio is reversed, suggest that maturational changes do occur.

The physiologic consequences of dopamine accumulation during hypoxia may be complex. In models of hypoxic-ischemic brain injury in both mature and perinatal animals, stimulation of striatal dopamine release acutely has been reported (Welch et al., 1978; Silverstein and Johnston, 1984; Brannan et al., 1987). 
In mature animals, dopamine nerve terminals are more susceptible to damage than serotonin nerve terminals in ischemic stroke (Weinberger and Cohen, 1983). Either directly, or indirectly in concert with excitatory amino acids, dopamine release acutely, postischemic dopaminergic hyperactivity, or both may contribute to the pathogenesis of neuronal injury (Globus et al., 1987). In 7-day-old rats that undergo right carotid artery ligation followed by exposure to $8 \%$ oxygen for 2 $\mathrm{h}$, the time threshold for onset of dopamine release in striatum ipsilateral to the carotid ligation coincides temporally with the threshold for irreversible injury. Based on the data presented here, it appears likely that the preceding period of hypoxia may augment the releasable dopamine pool.

Furthermore, during critical developmental periods, neurotransmitters may have specific trophic and regulatory roles. Prolonged periods of moderate hypoxic exposure in early postnatal rats $(12 \mathrm{~h} /$ day from days 2 to 10) produce long-lasting changes in dopamine metabolism and dopamine-related behaviors (Ihle et al., 1985; Lun et al., 1986). In the perinatal period, even subtle disruption of synaptic function resulting from transient hypoxia may have a significant impact on neuronal development.

Acknowledgment: This work was supported by grant NS01171 (to F. S. Silverstein). Dr. K. Gordon was the recipient of a fellowship from the Easter Seals Foundation of Canada.

\section{REFERENCES}

Benveniste H., Drejer J., Schousboe A., and Diemer N. H. (1987) Regional cerebral glucose phosphorylation and blood flow after insertion of a microdialysis fiber through the dorsal hippocampus in the rat. J. Neurochem. 49, 729-734.

Brannan R., Weinberger J., Knott P., Taff I., Kaufmann H., Togaski D., Nieves-Rosa J., and Maker H. (1987) Direct evidence of acute massive striatal dopamine release in gerbils with unilateral strokes. Stroke 18, 108-110.

Davis J. N. and Carlsson A. (1973) The effects of hypoxia on monoamine synthesis, levels, and metabolism in rat brain. $J$. Neurochem. 21, 783-790.

Globus M. Y. T., Ginsberg M. D., Dietrich W. D., Busto R., and Scheinberg P. (1987) Substantia nigra lesion protects against ischemic damage in the striatum. Neurosci. Lett. 80, 251-256.

Globus M. Y. T., Busto R., Dietrich W. D., Martinez E., Valdes I., and Ginsberg M. D. (1989) The role of dopamine and glutamate in the development of ischemic neuronal injury in the striatum, in Cerebrovascular Diseases (16th Research [Princeton] Conference on Cerebrovascular Diseases) (Ginsberg M. D. and Dietrich W. D., eds), pp. 99-107. Raven Press, New York.

Hedner T. and Lundborg P. (1979) Regional changes in monoamine synthesis in the developing rat brain during hypoxia. Acta Physiol. Scand. 106, 139-143.
Hedner T. and Lundborg P. (1980) Catecholamine metabolism in neonatal rat brain during asphyxia and recovery. Acta Physiol. Scand. 109, 169-175.

Ihle W., Gross J., and Moller R. (1985) Effect of chronic postnatal hypoxia on dopamine uptake by synaptosomes from striatum of adult rats. Biomed. Biochem. Acta 44, 433-437.

Lun A., Gross J., Beyer M., Fischer H. D., Wustmann C., Schmidt J., and Hecht K. (1986) The vulnerable period of perinatal hypoxia with regard to dopamine release and behavior in adult rats. Biomed. Biochem. Acta 45, 619-627.

Miwa S., Fujiwara M., Inoue M., and Fujiwara M. (1986) Effects of hypoxia on the activities of noradrenergic and dopaminergic neurons in the rat brain. $J$. Neurochem. 47, 63-69.

Robinson T. and Whishaw I. (1988) Normalization of extracellular dopamine in striatum following recovery from a partial unilateral 6-OHDA lesion of the substantia nigra: a microdialysis study in freely moving rats. Brain Res. 450, 209-224.

Roth R. H., Murrin L. I., and Walters J. R. (1976) Central dopaminergic neurons: effect of alterations of impulse flow on the accumulation of dihydroxyphenylacetic acid. Eur. J. Pharmacol. 36, 163-171.

Sharp T., Zetterstrom T., and Ungerstedt U. (1986) An in vivo study of dopamine release and metabolism in rat brain regions using intracerebral dialysis. $J$. Neurochem. 47, 113-122.

Silverstein F. S. and Johnston M. V. (1984) Effects of hypoxia-ischemia on monamine metabolism in the immature brain. Ann. Neurol. 15, 342-347.

Silverstein F., Buchanan K., and Johnston M. V. (1984) Pathogenesis of hypoxic-ischemic brain injury in a perinatal rodent model. Neurosci. Lett. 49, 271-277.

Silverstein F. S., Buchanan K. B., and Johnston M. V. (1986) Perinatal hypoxia-ischemia disrupts striatal high affinity $\left[{ }^{3} \mathrm{H}\right]$ glutamate uptake into synaptosomes. $J$. Neurochem. 47, 1614-1619.

Ungerstedt U. (1984) Measurement of neurotransmitter release by intracranial dialysis, in Measurement of Neurotransmitter Release In Vivo (Marsden C. A., ed), pp. 81-105. John Wiley and Sons, Chichester.

Vannucci R. C., Lyons D. T., and Vasta F. (1988) Regional cerebral blood flow during hypoxia-ischemia in immature rats. Stroke $19,245-250$.

Weinberger J. and Cohen G. (1983) Nerve terminal damage in cerebral ischemia: greater susceptibility of catecholamine nerve terminals relative to serotonin nerve terminals. Stroke 14,986 989.

Welch K. M. A., Wang T., and Chabi E. (1978) Ischemia-induced seizures and cortical monoamine levels. Ann. Neurol. 3, 152155.

Welsh F., Vanucci R., and Brierley J. (1982) Columnar alterations of NADH fluorescence during hypoxia ischemia in immature rat brain. J. Cereb. Blood Flow Metab. 2, 221-228.

Westerink B. H. C., Bosher F. J., and Wirix E. (1984) Formation and metabolism of dopamine in nine areas of the rat brain: modification by haloperidol. $J$. Neurochem. 42, 1321-1327.

Zaczek R. and Coyle J. T. (1982) Rapid and simple method for measuring biogenic amines and metabolites in brain homogenates by HPLC electrochemical detection. $J$. Neural Transm. 53, 1-5.

Zetterstrom T., Sharp T., Marsden C. A., and Ungerstedt U. (1983) In vivo measurement of dopamine and its metabolites by intracerebral dialysis: changes after $d$-amphetamine. $J$. Neurochem. 41, 1769-1773. 